tierischen Nahrungsmittel mit 9r,5\% gegen $8,5 \%$ der pflanzlichen.

Das gleiche Bild der Ernährung zeigt auch eine Berechnung der Ausgaben der Altonaer Arbeiterfamilie, gegliedert nach Calorien und dem in dem Nährwert enthaltenen Eiweiß, $F$ ett und Kohlenhydraten, die in Tabelle VI niedergelegt ist. des Normalcalorienbedarfs und 75,6\% des Eiweißbedarfs. Bei den I46 Familien der Julierhebung war ein weiterer Rückgang des Calorien- und Eiweißverbrauchs festzustellen. Der Calorienverbrauch sank auf 2232,2, der Eiweißverbrauch auf $66,7 \mathrm{~g}$ pro Tag und Kopf. In Prozenten ausgedrückt wurde vom Normalcalorienbedarf nur $82,7 \%$, vom Normal-

Tabelle VI.

Der Nahrungs- und Genußmittelverbrauch (in Calorien) einer Altonaer Arbeiterfamilie von 3 Köpfen 1920.

\begin{tabular}{|c|c|c|c|c|c|c|c|c|c|c|}
\hline \multirow{3}{*}{ Monate } & \multirow{3}{*}{\begin{tabular}{|c} 
Tatsächliche \\
Verbrauchsmenge \\
in Calorien
\end{tabular}} & \multicolumn{3}{|c|}{ Nährwert der Nahrungsmittel an } & \multirow{3}{*}{$\begin{array}{l}\text { Preis der Ver- } \\
\text { brauchsmenge } \\
\text { in Pfg. }\end{array}$} & \multirow{3}{*}{ Monate } & \multirow{3}{*}{$\begin{array}{l}\text { Tatsächliche Ver- } \\
\text { brauchsmenge } \\
\text { in Calorien }\end{array}$} & \multicolumn{3}{|c|}{ Nährwert der Nahrungsmittel an } \\
\hline & & EiweiB & Fett & $\begin{array}{l}\text { Kohlen- } \\
\text { hydraten }\end{array}$ & & & & Eiweiß & Fett & $\begin{array}{l}\text { Kohlen- } \\
\text { hydraten }\end{array}$ \\
\hline & & \multicolumn{3}{|c|}{ in $\mathrm{g}$} & & & & & & \\
\hline & \multicolumn{5}{|c|}{ Tatsächlicher Verbrauch in absoluten Zahlen } & \multicolumn{5}{|c|}{ Der Normalverbrauch jedes Monats ist gleich roo gesetzt } \\
\hline Januar . & I83394 & $43^{86}$ & $345^{6}$ & 32372 & $3237^{2}$ & Januar . . & 85,74 & $6 \mathrm{I}, 5 \mathrm{I}$ & 80,78 & $90,8 \mathrm{I}$ \\
\hline Februar . & I $4334^{\circ}$ & 3213 & $372 \mathrm{I}$ & 23332 & 32829 & Februar. . & $7 \pm, 63$ & 48,17 & 92,98 & 69,96 \\
\hline März . · · & I 88859 & $53+3$ & 3549 & $3247^{6}$ & $45 \circ 8 \mathrm{I}$ & März . & 88,29 & $74,5^{2}$ & 82,96 & $9 I$, ro \\
\hline April . . . & I68 330 & $4 \times 75$ & $4^{6} 3 I$ & 26213 & 43968 & April. & $8 I, 32$ & $60,5 \mathrm{r}$ & I I I, 86 & 75,98 \\
\hline $\mathrm{Mai}$. & I 81124 & 4202 & 4005 & 30147 & 50968 & Mai & 84,68 & $5^{8,93}$ & 93,62 & 84,56 \\
\hline Juni . . & I8592i & 4128 & 5447 & 28684 & 53936 & Juni . & 83,30 & $55,4^{8}$ & $\mathrm{I} 22,02$ & $77, \mathrm{II}$ \\
\hline Juli . & 147164 & $33^{84}$ & 4292 & $2285 \mathrm{I}$ & 46493 & Juli : & 68,80 & 47,46 & $\pm 00,33$ & 64, ro \\
\hline August . . & I 85877 & 4327 & $470 \mathrm{I}$ & 30200 & 53190 & August . & 86,90 & 60,69 & IO9,89 & $84,7 \mathrm{I}$ \\
\hline September & 204866 & $455^{\circ}$ & $574^{8}$ & 32432 & 52443 & September & 98,97 & 65,94 & $I 3^{8,84}$ & 94, or \\
\hline Oktober . & 213110 & 5215 & 6429 & 32123 & $5^{8} \mathrm{O}^{\circ}$ & Oktober & 99,63 & 73,14 & $I 5^{0,28}$ & 90, I I \\
\hline November & I68 720 & 3605 & 4040 & 28692 & 48516 & November. . & $8 I, 5 I$ & 52,25 & 97,58 & 83,17 \\
\hline Dezember & 216060 & 47 I I & 6333 & 33684 & 88 I. 8 & Dezember & IOI,OI & 66,07 & $I_{4} 8,04$ & 94,49 \\
\hline
\end{tabular}

Setzt man den Normalverbrauch dieser dreiköpfigen Arbeiterfamilie in einem jeden Monat gleich roo, so ergibt sich die Tatsache, daß bis auf wenige Monate niemals der Normalverbrauch, und zwar sowohl bezüglich der Ausnutzungszahlen (Calorien) wie auch der Zusammensetzung der Nahrungsmittel nach Eiweiß, Fett und Kohlenhydraten erreicht worden ist. Der Calorienverbrauch wird annähernd erreicht in den Monaten September $(98,97)$ und Oktober $(99,63)$; im Dezember mit IOI,OI - eine Folge des Weihnachtsfestes und der Neujahrsfeier - sogar überschritten. Der kleinste Calorienverbrauch ergibt sich im Juli mit 68,80. Die Normalmenge an Eiwei $\beta$ und Kohlenhydraten wird in keinem Monat erreicht. Besonders groß ist auch hier der Mangel an Eiweiß; so wird in den Monaten Februar und Juli nicht einmal die Hälfte, mit Ausnahme der Monate März und Oktober in keinem Monat $2 / 3$ des Normalverbrauchs gedeckt. Wesentlich besser steht es mit den Kohlenhydraten; durchschnittlich werden $4 / 5$ des Normalverbrauchs konsumiert. Eine eigenartige Entwicklung zeigt der tatsächliche Fettverbrauch. Bis auf die Monate Januar, Februar, März, Mai und November, in denen der Normalverbrauch nicht ganz, aber doch stets über $4 / 5$ erreicht wird, ist in den einzelnen Monaten mehr, z. T. sogar bis 50\% mehr als der Normalverbrauch konsumiert worden. Diese Tatsache wird aller Wahrscheinlichkeit nach mit der Aufhebung der $Z_{\text {wangsbewirtschaftung }}$ der Fette und dem Verlangen nach der so stark entbehrten fettreichen Nahrung im Zusammenhang stehen.

Es ist nun äußerst interessant, diese Ergebnisse mit Untersuchungen über die Ernährung während des Krieges in der Zeit, in der die Nahrungsmittel rationiert waren, vergleichen zu können. Eine solche Untersuchung ist in dankenswerter Weise von Prof. Dr. LoEwy auf Grund der von mir bearbeiteten Lebenshaltungserhebungen des Kriegsausschusses für Konsumenteninteressen im April und Juli I9r6 durchgeführt worden. Seine Ergebnisse sind niedergelegt in dem in der ,Deutschen Medizinischen Wochenschrift" erschienenen Aufsatz: „Über Kriegskost"1). LOEWY kommt zu dem Resultat, daß auch bei den im April erhobenen 858, ebenso wie bei den im Juli erhobenen $\mathrm{r}_{4} 6$ Familien sowohl der Calorienbedarf als besonders der Eiweißverbrauch hinter dem Normalverbrauch zurückgeblieben ist. Denn pro Tag und Kopf (Kopf gleich Konsumtionseinheit unter Berücksichtigung des Minderverbrauchs der Kinder) stellte sich die Calorienmenge bei den 858 Familien der Aprilerhebung auf 2320, die Eiweißaufnahme auf $68,3 \mathrm{~g}$; in Prozenten ausgedrückt also $85,9 \%$

\footnotetext{
1) Jahrg. I9I7, Nr. 6 u. ל.
}

eiweißbedarf nur 74,2\% gedeckt. Vergleicht man dieses Ergebnis mit den vorliegenden, nach dem Kriege erhobenen Verbrauchsberechnungen, so zeigt sich eine große Übereinstimmung. Entgegen der vielfach geäußerten Meinung ist durch die Aufhebung der Blockade infolge der starken Preissteigerung die Ernährung weiter Volksschichten gegenüber der Kriegszeit keine wesentlich bessere geworden. Die Verschlechterung der Valuta, die die Wirkung hatte, gerade die notwendigsten Lebensmittel im Preise stark zu steigern, hat zur Folge gehabt, daß auch gegenwärtig die minderbemittelten Schichten durch ihre Ernährung nicht den vollen Calorienbedarf decken, ganz besonders aber nicht so viel Eiweiß aufnehmen, wie notwendig wäre, um voll leistungsfähig zu sein.

\section{UNTERSUCHUNGEN ÜBER DAS CHINIDIN, SEINE ANTAGONISTEN UND SYNERGISTEN.}

Von

Dr. ERNST WIECHMANN,

Assistent der I. Međizin. Klinik der Universität München. (Direktor: Prof, Dr. Ernst von Romberg.)

Im Jahre I9I 8 berichtete W. FREY ${ }^{1}$ ) über I2 Fälle von Arhythmia perpetua, von denen 7 durch Chinidin regelmäßig geworden waren. Seitdem gibt es eine Chinidintherapie des Vorhofflimmerns. Heute, genau 4 Jahre später, ist das Chinidin von den verschiedensten Seiten im In- und Auslande zur Beseitigung der Arhythmia perpetua verwandt worden*). Abschließend läßt sich sagen, daß sich in etwa $5^{\circ} \%$ der Fälle mit Chinidin eine Regularisierung der Herztätigkeit erzielen läßt, daß die Regularisierung aber meist nicht dauernd anhält. Trotz dieser nicht gerade glänzenden Erfolge ist das Chinidin dennoch als eine wertvolle Bereicherung des uns zur Bekämpfung der perpetuellen Arhythmie zur Verfügung stehenden Arzneischatzes anzusprechen. Denn auch eine nur zeitweise Aufhebung des Flimmerzustandes der Vorhöfe ist für den Kreislauf günstig.

Keinesfalls ist jedoch das Chinidin als ein harmloses, gar etwa ohne weiteres für die ambulante Praxis geeignetes Mittel zu bezeichnen. Dazu sind die bei seiner Anwendung zwar nur selten in Erscheinung tretenden, indes nicht auszuschließenden Gefahren zu groß.

*) Die Ergebnisse unserer Klinik sind durch BENJAMIN und VON KAPFF zusammengestellt worden (vgl. BENJAMIN und VON KAPFF, Dtseh. med. Wochenschr I92I, Nr. I; ferner VON KAPFF, Dtsch. med. Wochenschr. I922, Nr. I4). 
Zunächst die Möglichkeit der Loslösung von Thromben. Vorhöfe, die sich im Zustand des Flimmerns befinden, sind meist erweitert, und dabei ist die Thrombenbildung recht häufig. Werden die flimmernden Vorhöfe durch Chinidin zum regelmäßigen Schlagen gebracht, so kann sich ein Thrombus loslösen und zu unangenehmen Komplikationen, unter Umständen zum Tode führen. Gegen diese Möglichkeit wird sich der Arzt nie schützen können. Jedoch hat dies das Chinidin mit der Digitalis gemeinsam; denn auch die Digitalis kann, wenn sie zur Beseitigung der Arhythmia perpetua angewandt wird, Ursache der gleichen Ereignisse sein.

Die größte Gefahr bei der ganzen Chinidintherapie ist offenbar durch die unterschiedliche Empfindlichkeit der einzelnen Individuen gegen Chinidin gegeben. Sie wird allen, die Chinidin verordnet haben, schon aufgefallen sein; sie ist auch, wie ich experimentell gezeigt habe, am Kaitblüterherzen vorhanden. Auf ihr Konto sind sicherlich in erster Linie die vereinzelt bei der Chinidinbehandlung des Vorhofflimmerns beobachteten schweren $Z$ uistände $z u$ setzen, die in der Literatur beschrieben sind (FREY ${ }^{2}$ ), BOCK ${ }^{3}$ ), $\mathrm{HAGEN}^{4}$ ), HAASS $\left.\left.^{5}\right)\right]$. Auch die bereits in den Jahren I 878 und 1880 von STRÜMPELL ${ }^{6}$ ) und FREUDENBERGER ${ }^{7}$ ) mitgeteilten, im Anschluß an eine Anwendung des Chinidins bei anderen Krankheiten erfolgten Todesfälle sind vermutlich durch Idiosynkrasie bedingt. Alle diese Zustände imponieren klinisch als eine schwere Schädigung des Atemzentrums. Mit der Atmung scheint aber immer gleichzeitig die Zirkulation stark geschädigt (FREY). Neuerdings hat W. FREY ${ }^{2}$ ) deswegen experimentell am Kaninchen festzustellen gesucht, wo bei toxischen Chinidinwirkungen der primäre Angriffspunkt ist. Es zeigte sich, daß das Chinidin in erster Linie die Herztätigkeit lähmt und erst hinterher die Atmung stillstellt.

Im Mittelpunkt der toxischen Chinidinwirkung steht also die Herzlähmung. Mit dieser wichtigen Feststellung erhebt sich sofort die Frage, ob es möglich ist, den Gefahren der Chinidinschädigung zu begegnen. Auch hicr heißt es, wie immer in der Therapie, daß das Vermeiden von Schädigungen viel wichtiger ist als die Beseitigung nach ihrem Eintritt. Schädigungen kann man vermeiden, wenn man zu Beginn der Chinidintherapie tastend vorgeht und vor allem nur kompensierte Herzen mit Chinidin angeht. Mit von BERGMANN ${ }^{8}$ ) geben wir an unserer Klinik am ersten Tage abends 0,2 Chinidin. sulfur. Treten nach dieser probatorischen Dosis irgendwelche Beschwerden, wie Brechneigung, Durchfälle, Schwindel, Ohrensausen u. a. m. auf, so sehen wir von einer weiteren Chinidinmedikation ab. $\mathrm{Da}$ wir von dem Chinin wissen ${ }^{9}$ ), da $\beta$ die Menstruation einen Einfluß auf das Entstehen von Chininnebenwirkungen haben kann, wird es sich empfehlen, möglichst auch kein Chinidin während der Menstruation zu geben. Aus dem Gesagten ergibt sich ohne weiteres, daß eine intravenöse Chinidintherapie - die Firma Kahlbaum bringt Ampullen von Chinidin. lactic, in den Handel vollkommen zu verwerfen ist. Zur Vermeidung von Nebenwirkungen wird es auch dienen, wenn man prinzipiell das Chinidin. sulfur. verwendet, das, wie ich ${ }^{10}$ ) früher zeigen konnte, im Vergleich zum Chinidin. pur. entsprechend seiner besseren Löslichkeit weit gründlicher resorbiert wird. Will man dennoch das Chinidin. pur., und zwar in etwa der gleichen Dosierung wie das Chinidin. sulfur., verwenden, so muß man zum mindesten in allen Fällen, wo die Säuremenge im Magen herabgesetzt ist, z. B. im Fieber, zu gleicher Zeit dem Magen Säure zuführen. Tut man das nicht, so schafft man unnötigerweise unübersichtliche Verhältnisse. Zur Klarheit des therapeutischen Bildes trägt es auch bei, wenn man prinzipiell kein weiteres Medikament neben dem Chinidin anwendet.

Treten trotz aller dieser Vorsichtsmaßregeln akute toxische Schädigungen des Herzens auf, so gilt es schnell zu handeln. Wie man hierbei evtl. versuchen muB vorzugehen, darauf weisen Versuche am isolierten Froschherzen hin, zu denen ich ${ }^{11}$ ) kürzlich in ganz anderem Zusammenhang geführt wurde. Wenn ich bei einem spontan in Ringerlösung schlagenden, nach AMSLER ${ }^{12}$ ) aufgeschlitzten Temporarienherzen die Ringerlösung gegen eine Ringerlọsung mit einem Zusatz von
$0,06 \%$ bis $0,2 \%$ Chinidin. sulfur. anstauschte, so trat meist in wenigen Minuten Lähmung des Herzens ein. Diesen Herzstillstand habe ich durch Calciumchlorid stets mehr oder weniger weitgehend beseitigt. Eine ähnliche Wirkung wie das Calciumchlorid übten Strontiumchlorid und Bariumchlorid aus. Aus theoretischem Interesse habe ich auch untersucht, ob die Chloride anderer mehrwertiger Kationen, wie $\mathrm{MgCl}_{2}, \mathrm{CoCl}_{2}$, $\mathrm{MnCl}_{2}, \mathrm{NiCl}_{2}$ und $\left[\mathrm{Co}\left(\mathrm{NH}_{3}\right)_{8}\right] \mathrm{Cl}_{3}$ dieselbe Fähigkeit besitzen wie das Calciumchlorid. Aber das war nicht der Fall. Diese für das Calciumchlorid festgestellte Tatsache kann in Parallele gesetzt werden zu der Beobachtung ZoNDEKs ${ }^{13}$ ), daß das durch Chinin gelähmte Herz durch Calcium wieder zum Schlagen gebracht wird, und ferner $z u$ jener von BoDEN und NEUKIRCH ${ }^{14}$ ), daß die Wirkung des Chinidins auf das Elektrokardiogramm des isolierten Kaninchenherzens durch Zusatz von Calcium zur Nährlösung kompensiert wird. Wenn es FREY ${ }^{2}$ ) nicht geglückt ist, am intakten Kaninchen durch vorangehende intravenöse Injektion von Calc. chlorat. den Herzstillstand nach intravenöser Chinidininjektion $\mathrm{zu}$ verhindern oder wenigstens zu verzögern, so mag das an der Dosierung liegen.

Klinisches Interesse erforderte es, weiterhin festzustellen, ob die Digitaliskörper auf das durch Chinidin vergiftete Froschherz eine ähnliche Wirkung wie Calcium ausüben. Das ist tatsächlich der Fall und war auch, nachdem ZoNDEK ${ }^{13}$ ) festgestellt hat, daß die Chininwirkung auf das Froschherz durch Strophanthin kompensiert wird, nicht anders zu erwarten. Ich habe $0,00016 \%$ Strophanthin oder $0,0009 \%$ Digifotin (in wässeriger Lösung) zur Chinidin-Ringerlösung hinzugesetzt. Immer wurde das stillgestellte Froschherz dadurch wieder zum Schlagen gebracht. Wässeriges Digitalisinfus in einer der verwandten Digifolinmenge entsprechenden Dosierung vermag dieses nicht $z u$ bewirken, auch wenn es wegen der bekanntlich schon nach 24 Stunden um die Hälfte herabgesetzten Wirksamkeit frisch verwandt wird. Ist das die Folge des im Infus fehlenden Digitoxins? Zur Entscheidung dieser Frage habe ich untersucht, ob das durch Chinidin gelähmte Froschherz nach Zusatz von $0,0000865 \%$ bis o,00I $73 \%$ Digitoxin*) zum Chinidin-Ringergemisch wieder zu schlagen beginnt. Das gelang nicht. Da jedoch Digitoxin in Wasser nicht löslich ist und deswegen in $44 \%$ Spiritus vini gelöst werden mußte, ist es nicht ausgeschlossen, daß der negative Ausfall dieser Versuche durch das alkoholische Lösungsmittel bedingt ist. Unentschieden bleibt es, ob in den beschriebenen Versuchen die Wirkung der Digitaliskörper in einer Sensibilisierung des Herzens durch Calcium besteht (LöwI ${ }^{15}$ ), oder ob Digitaliskörper und Calcium dabei gleichsinnig wirken [ZoNDEK $\left.{ }^{13}\right)$ ], oder ob es sich schlieBlich dabei um eine katalytische Wirkung des Calciums handelt [WIEchmanN ${ }^{16}$ )].

Für die Klinik ergibt sich aus meinen Versuchen die Forderung, wenn es einmal zu akuten Chinidinschädigungen des Herzens kommen sollie, ihre Beseitigung durch intravenöse Injeltion von Strophanthin oder Calcium oder evtl. auch durch subcutane Injeltion von Digifolin zu versuchen. Diese Forderung ist berechtigt, auch wenn meine Versuche nur am isolierten Froschherzen ausgeführt wurden. Waren doch jene bekannten Versuche SANTESSONS ${ }^{17}$ ) über die Wirkung der Chinaalkaloide auf das Froschherz der Ausgangspunkt der ganzen Chinidintherapie.

Das souveräne Mittel bei jenen oben geschilderten Zustönden wird wohl immer das Strophanthin sein. Man wird es stets unbesorgt anwenden können, wenn man nach dem Grundsatz unserer Klinik nur kompensierte Herzen mit Chinidin angeht und zwischen dem Absetzen der Digitalis- und dem Beginn der Chinidinmedikation einen Zeitraum von Io-I4 Tagen einschiebt, da dann die Kombination des Strophanthins mit der Digitalis zu einer gefahrvollen Überdosierung nicht vorhanden sein kann. Vor einer gleichzeitigen Verabreichung von Strophanthin und Calcium ist dringend $z u$ warnen. Hierin bin ich mit $O$. Löwr ${ }^{18}$ ) einer Ansicht. Ob das Strontiumchlorid zur intravenösen Injektion beim Menschen geeignet

*) Digifolin und Digitoxin wurden mir liebenswürdigerweise von der Gesellschaft für chemische Industrie in Basel, Strophanthin von der Firma C. F. Böhringer in Mannheim zur Verfügung gestellt. 
ist, vermag ich nicht zu sagen. Vielleicht geben uns die von BorUtTau und Grasshem ${ }^{19}$ ) in Aussicht gestellten Untersuchungen über die Pharmakologie des Strontiums hierüber Aufschluß.

Damit sind eine Reihe von Antagonisten des Chinidins besprochen. Gibt es auch Synergisten? Schon rgI9 empfahl VON BERGMANN ${ }^{8}$ ) die Kombination der Chinidinmedikation mit einer Kalianreicherung des Organismus. Die experimentelle Grundlage fehlte bisher hierfür. Ich konnte sie liefern: Wird der Kaliumgehalt der Ringerlösung um eine an sich nicht wirksame Menge erhöht, so sind schon sonst sicher unterschwellige Chinidindosen imstande, das Froschherz zu lähmen. Für die therapeutische Verwendung könnte man aus diesem Ergebnis folgern, daß gleichzeitige Kaliumzufuhr eine Herabsetzung der Chinidindosis ermöglicht. Bei der praktischen Verwirklichung dieser Folgerung ergeben sich jedoch Schwierigkeiten. Zunächst müssen, um eine Kalianreicherung 'des Körpers zu erzielen, große Kaliummengen zugeführt werden. VoN BergmanN ${ }^{8}$ ) hat deswegen empfohlen, die Chinidinkur mit kalireicher Kost (Kartoffeln) zu kombinieren. $F_{R E Y^{20}}$ ) hat dagegen, wohl mit einem gewissen Recht, eingewandt, daß der mit Kohlenhydraten überlastete Darm zum Meteorismus neigen wird, was bei drohenden Dekompensationszuständen nicht angenehm ist. Dem Einwand Freys gegenüber von BergmanN, daß die Möglichkeit einer Anreicherung des Herzens mit Kalisalzen bisher nicht nachgewiesen ist, vermag ich nicht beizustimmen, denn die Giftwirkung von Kalisalzen vornehmlich auf das Herz ist doch altbekannt. Ferner ist zu bedenken, daß jeder, der sich kalireich ernährt, zwangsmäßig instinktiv (G. vON BUNGE) ein großes Kochsalzbedürfnis hat. $O b$ hier allerdings nicht geschmackliche Gründe eine Rolle spielen, erscheint fraglich"1). Vielleicht liegt die Wahrheit in der Mitte. Da übermäßige Kochsalzzufuhr bei allen Hypertonien zu vermeiden ist ${ }^{22}$ ), und die ungünstige Einwirkung von Kalisalzen auf Nierenschädigungen bekannt ist, scheidet somit eine Reihe von perpetuellen Arhythmien für die Kalibehandlung aus. Bei Herzkranken, die zu Ödemen neigen, wird man sich dagegen von einer Kalizufuhr nur Gutes versprechen können. Es sei hier an das alte Diureticum Kalium aceticum erinnert. Erwägt man dies alles, so wird es sich empfehlen, bei allen perpetuellen Arhythmien, die weder eine Hypertonie über $160 \mathrm{~mm}$ $\mathrm{Hg}$ noch eine nicht arteriosklerotisch bedingte Nierenschädigung haben, eine Unterstützung der Chinidintherapie durch einen passend zusammengestellten kalireichen Speisezettel evti. zusammen mit oraler Darreichung von Kalium chloratum (Lösung 5:I50, eßlöffelweise) zu versuchen. Als besonders kalireich seien folgende Nahrungs- und Genußmittel ${ }^{23}$ ) genannt:

\begin{tabular}{|c|c|c|}
\hline soo $g$ enthalten & & $\mathrm{mg} \mathrm{K}_{2} \mathrm{O}$ : \\
\hline Liebigs Fleischextrakt & . & 8800 \\
\hline Kakao, aufgeschlossen & . & 4170 \\
\hline Schellfisch, gesalzen & . . & 1570 \\
\hline Bohnen, weiße... &. & 1300 \\
\hline Mandeln, süße ... & . . & 1280 \\
\hline Kakao, gewöhnlicher . & . . & 1250 \\
\hline Rettich & . . & 1200 \\
\hline Feigen, getrocknet . . & . . & I I 60 \\
\hline Knorrs Erbsmehl . . & . . & I 100 \\
\hline Trüffel . . . . & . . & 1080 \\
\hline Erbsen. . . & . . & 980 \\
\hline Rindfleisch, mittelfett & & 870 \\
\hline Meiereikäse . . . . & . . & 620 \\
\hline Steinpilze . & . . & $6 \mathrm{IO}$ \\
\hline Kartoffel . & . . & 580 \\
\hline Hubn, Fleisch, fett . & . . & 560 \\
\hline Simonsbrot . . . & . . & 530 \\
\hline Kalbfleisch . . . & . . & 520 \\
\hline Aprikosen . . . . & . . & 490 \\
\hline Savoyerkohl (Wirsing) & . & 460 \\
\hline Endiviensalat . . & . & 460 \\
\hline Weintrauben & . . & 460 \\
\hline Rosenkohl. . & . . & 450 \\
\hline Bananen . : . . & 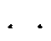 & 435 \\
\hline Schellfisch, frisch. . & . & 400 \\
\hline Himbeersaft . . . . &. & 130 \\
\hline
\end{tabular}

Bei der Auswahl aus dieser Tabelle wird man selbstverständlich individuellen Neigungen und finanziellen Verhältnissen Rechnung tragen müssen. Will man aus den oben angeführten Gründen ein Salzen der Kartoffeln vermeiden, so empfiehlt es sich, nach von NoORDEN-SALOMON ${ }^{24}$ ), die Kartoffeln mit der Schale in heißer Asche zu rösten und mit Butter zu verspeisen, oder aber durch Beigabe einfacher unschädlicher Gewürze, wie Suppengrün, Tomatentunke, Rahm- und Buttertunke, das Salz entbehrlich zu machen.

Als Beispiel einer kalireichen Kostform führe ich an: $200 \mathrm{~g}$ mittelfettes Rindfleisch, 750 g Milchkakao, $600 \mathrm{~g}$ Kartoffeln, I $5^{\circ} \mathrm{g}$ Simonsbrot, $25^{\circ} \mathrm{g}$ Rosenkohl, I ganzes Ei, $5^{\circ} \mathrm{g}$ ungesalzene Butter, roo $\mathrm{g}$ Feigen, $5 \mathrm{~g}$ Liebigs Fleischextrakt. Wert dieser Kost $=$ Io7 $\mathrm{g}$ Eiweiß, I2,8 $\mathrm{g} \quad \mathrm{K}_{\mathbf{2}} \mathrm{O}$, $27^{8} 3$ Calorien.

Gibt man hierzu noch Kalium chloratum per os und rechnet man mit URBEANU ${ }^{25}$ ) 4-5 g Kaliumoxyd als physiologisches Kaliumminimum für die Tagesration, so ist der Wert von fast $\mathrm{I}_{3} \mathrm{~g} \mathrm{~K}_{2} \mathrm{O}$ in der angegebenen Kostform sehr hoch. Der von URBEANU für Deutschland pro Kopf und Tag angegebene Wert von $\mathrm{I}_{3} \mathrm{~g} \mathrm{~K}_{2} \mathrm{O}$ (Kartoffeln!) ist, vor allem für Süddeutschland, viel zu groß.

Vorläufig scheint mir die Bedeutung der Kalium-Chinidinversuche viel mehr auf theoretischem als auf praktisch-klinischem Gebiet zu liegen. Wie schon für das Strophanthin durch LöwI, für das Chinin durch ZoNDEk, ist hier für das Chinidin ein Zusammenhang zwischen der Wirkung organischer Arzneimittel und anorganischem Kation dargetan. Sicherlich werden solche Zusammenhänge noch viel mehr bestehen, als wir heute vielleicht vermuten.

Literatur: 1) W. FReY, Berl. klin. Wochenschr. IgI8, Nr. I8, I9, 36. - ${ }^{2}$ ) FREY u. HAGEManN, Zeitschr. f. d. ges. exp. Med. 25, 290. 1921. ${ }^{3}$ ) Bock, Med. Klin. I92I, Nr. 35. 4). HAGEN, Wissenschaftlich-medizinischer Verein in Köln, 2. VI. I921 (vgl. Dtsch. med. Wochenschr. I92I, Nr. 39). - ${ }^{5}$ ) HaAss,
Berl. klin. Wochenschr. I92I, Nr. 21. ${ }^{6}$ ) STRÜMPEL, Berl. klin. Wochenschr. I878, Nr. 46. - 7) Freudenberger, Dtsch. Arch. f. klin. Med. 26, 577. I880. $-{ }^{8}$ ) von BergmanN, Münch. med. Wochenschr. I919, Nr. 26. - 9) LEwiN, Nebenwirkungen der Arzneimittel. Berlin I899. - 10) E. Wiechmann, Zeitschr. f. d. ges. exp. Med. 7, I55. I9I8. - 11) E. Wifchmann, Pflügers Arch. f. d. ges. Physiol. 195, 588, 1922. - 12) AMSLER, Zentralbl. f. Physiol. 31, 467. I91 7. --. 13) ZoNDEK, Dtsch. med. Wochenschr. I921, Nr. 30. - $\left.{ }^{14}\right)$ BODEN u. NEUKIRCH, Dtsch. Arch. f. klin. Med. 136, I8I. 192 I. - 15) O. LöwI, Arch. f. exp. Pathol. u. Pharmakol. 82, I 31. I917, und 83, 366. 1918. Vgl. ferner A. von KonschegG, Arch. f. exp. Pathol. u. Pharmakol. 7I, 25 I. I9I3. 16) E. Wiechmann, Pflügers Arch. $f$. d. ges. Physiol. 194, 435. 1922. - 17) SAntesson, Arch. f. exp. Pathol. u. Pharmakol. 32, 321. I 893. - ${ }^{18}$ ) O. LöwI, Arch. f. exp. Pathol. u. Pharmakol. 82, I 3 I. r918. - ${ }^{19}$ ) Boruttau u. Grassheim, Zeitschr. f. d. ges. exp. Med. 27, $213.1922 .-{ }^{20}$ ) FREY, Therap. Halbmonatshefte 35, 534. r92I. - 21) Vgl. hierzu von NoORDEN-SALOMON, Handb. d. Ernährungslehre Bd. I. Berlin I920. $-{ }^{22}$ ) Vgl. hierzu voN RoMBERG, Krankheiten des Herzens und der Blutgefäße. Stuttgart I92 I. - ${ }^{23}$ ) Nach der Nahrungsmitteltabelle von ScHALL-HeIster, Würzburg I9I7. - 24) voN NooRdEN-SALOMON, Handb. d. Ernährungslehre, Bd. I. Berlin I920. - ${ }^{25}$ ) URBEANo, Die Gefahr einer an Kaliumverbindungen zu armen Ernährungsweise und ihre Beziehung au Ernährungskrankheiten. Berlin-Wien 1916.

\section{EXPERIMENTELI NACHWEISBARE ZUSAMMEN- HÄNGE ZWISCHEN DEM VIRUS DER ENCEPHA- LITIS EPIDEMICA UND DEMJENIGEN DES HERPES FEBRILIS.}

Von

Dr. Alfred SchNabEL.

Aus dem Institut für Infektionskrankheiten „Robert Koch“ in Berlin.

Je weiter die Zeit zurückliegt, in der zuletzt die sog. Encephalitis epidemica gehäuft auftrat, desto mehr erlischt begreiflicherweise das Allgemeininteresse für diese Erkrankung. Aber gerade die Erfahrungen der letzten Epidemie, welche im Zusammenhang mit den sich anschließenden experimentellen Untersuchungen die Spezifizierung dieser Krank- 